\title{
Pushing the Limits of OWL 2 Reasoners in Ontology Alignment Repair Problems
}

\author{
Alessandro Solimando@* , Ernesto Jiménez-Ruiz ${ }^{\natural}$ and Giovanna Guerrini ${ }^{\circledR}$ \\ ${ }^{a}$ Dipartimento di Informatica, Bioingegneria, Robotica e Ingegneria dei Sistemi, \\ Università di Genova, Via Dodecaneso 35, 16146, Italy \\ E-mail: alessandro.solimando@unige.it,giovanna.guerrini@unige.it \\ ${ }^{\mathrm{b}}$ Department of Computer Science, \\ University of Oxford, Wolfson Building, Parks Road, Oxford OX1 3QD, UK \\ E-mail: ernesto.jimenez-ruiz@cs.ox.ac.uk
}

\begin{abstract}
Ontologies play a key role in the development of the Semantic Web and are being used in many diverse application domains such as biomedicine and e-commerce. An application domain may have been modeled according to different points of view and purposes. This situation usually leads to the development of different ontologies that intuitively overlap, but that use different naming and modeling conventions. The problem of (semi-)automatically integrating independently developed ontologies through mappings, is usually referred to as the ontology matching problem. Ontology matching systems, however, rely on lexical and structural heuristics, and the integration of the input ontologies and the mappings may lead to many undesired logical consequences, which could sensibly diminish their usefulness. The present paper, on the one hand aims at veryfing the hypothesis that classification of large ontologies via mappings still poses a challenge to OWL 2 reasoners. On the other it also explores the applicability of OWL 2 reasoning for the repair of unintended entailments (namely, unsatisfiable concepts or violations of the conservativity principle). In this paper we provide an update on the feasibility of using OWL 2 reasoners to repair the integration of ontologies via mappings, providing a more accurate evaluation of the feasibility of extracting all the justifications. Additionally, the current evaluation also encompasses the analysis of the use of OWL 2 reasoners for solving the violations of the so-called conservativity principle.
\end{abstract}

Keywords: Reasoning, Ontology Matching, Ontology Alignment Debugging, Ontology-based Data Integration

\section{Introduction}

The problem of (semi-)automatically computing mappings between independently developed ontologies is usually referred to as the ontology matching problem. A number of sophisticated ontology matching systems have been developed in the last years [829]. Ontology matching systems, however, rely on lexical and structural heuristics and the integration of the input ontologies and the mappings may lead to many undesired logical consequences (e.g., unsatisfi-

*Corresponding author. E-mail: alessandro.solimando@unige.it Tel: +39-010-353-6616. Fax: +39-010-353-6699. able classes or violations of the conservativity principle).

The fix of undesired logical consequences caused by ontology mappings is known as the mapping repair problem [14]. Mapping repair can be addressed using state-of-the-art approaches for debugging OWL 2 ontologies, which rely on the extraction of justifications for the unwanted axiom (e.g., [13 15[27|34]). However, in [12] it was pointed out that justificationbased technologies do not scale when the number of such axioms is large (a typical scenario in mapping repair problems).

This paper extends our previous evaluations presented in [1232], in different respects. [12] provided a first evaluation on the use of OWL 2 reasoning for 
the classification and repair of integrated ontologies. [32] also considered reasoners for OWL 2 profiles (i.e., $E L K)$, and tested the extraction of more than a single justification for computing a repair for logical violations represented by incoherent classes.

Our extended evaluation is based on the datasets and ontology matching systems from the Ontology Alignment Evaluation Initiative (OAEI) [8]. In addition to the previous versions of the evaluation, in this paper we also: (i) consider the so-called violations of the conservativity principle, that is, novel axioms entailed by the aligned ontology, involving elements of one of the two input ontologies, that are not entailed by the input ontologies in isolation [131]. (ii) provide extended experimental results concerning the extraction of (a subset of) all the justifications for a given entailment, providing additional insights on the problem. (iii) compare the black-box justification extraction techniques with one of the latest glass-box approaches based on tracing, for the optimization of the justifications extraction.

Our results suggest that the classification of the integration of large ontologies via mappings still poses a challenge to OWL 2 reasoners. Furthermore, the repair of unintended entailments (e.g., unsatisfiable concepts or conservativity violations) using OWL 2 reasoners critically compromises the performance of mapping repair systems in the best case, or it is simply not tractable when all the justifications need to be extracted in order to compute an optimal repair.

The remainder of the paper is organised as follows: Section 2 introduces the needed preliminaries, Section 3 describes the dataset, the environment used for the evaluation, and also provides and discusses in detail its results. Finally, Section 4 concludes the paper.

\section{Preliminaries}

In this section, we present the formal representation of ontology mappings (Section 2.1), the notions of semantic difference (Section 2.2), mapping coherence and conservativity principle violations (Section 2.3).

\subsection{Representation of Ontology Mappings}

Mappings are conceptualised as 4-tuples of the form $\left\langle e_{1}, e_{2}, n, \rho\right\rangle$, where $e_{1}, e_{2}$ are entities in the vocabulary or signature of the relevant input ontologies $\mathcal{O}_{1}$ and $\mathcal{O}_{2}$ (i.e., $e_{1} \in \operatorname{Sig}\left(\mathcal{O}_{1}\right)$ and $e_{2} \in \operatorname{Sig}\left(\mathcal{O}_{2}\right)$ ), $n$ is a confidence measure between 0 and 1 , and $\rho$ is a re- lationship between $e_{1}$ and $e_{2}$, typically subsumption (i.e., $e_{1}$ is more specific than $e_{2}$ ), equivalence (i.e., $e_{1}$ and $e_{2}$ are synonyms) or disjointness (i.e., $e_{1}$ and $e_{2}$ cannot share individuals) [7].

RDF Alignment [5] is the main format used in the Ontology Alignment Evaluation Initiative (OAEI) to represent mappings containing the aforementioned elements. Additionally, mappings are also represented as OWL 2 subclass, equivalence, and disjointness axioms [4]; mapping confidence values $(n)$ are then represented as axiom annotations. Such a representation enables the reuse of the extensive range of OWL 2 reasoning infrastructure that is currently available. Note that alternative formal semantics for ontology mappings have been proposed in the literature (e.g., $[2723]$ ), but they are out of scope of the present article because the reasoning in the aligned ontology cannot be achieved directly with OWL 2 reasoning infrastructure, rather it requires custom reasoning facilities.

\subsection{Semantic Consequences of the Integration}

The ontology resulting from the integration of the two ontologies $\mathcal{O}_{1}$ and $\mathcal{O}_{2}$ via a set of mappings $\mathcal{M}$ typically entails axioms that do not follow from $\mathcal{O}_{1}$, $\mathcal{O}_{2}$, or $\mathcal{M}$ alone. These new semantic consequences can be captured by the notion of deductive difference [18 19].

Intuitively, the deductive difference between $\mathcal{O}$ and $\mathcal{O}^{\prime}$ w.r.t. a signature $\Sigma$ is the set of entailments constructed over $\Sigma$ that do not hold in $\mathcal{O}$, but do hold in $\mathcal{O}^{\prime}$. No algorithm is available for computing the deductive difference for DLs more expressive than $\mathcal{E} \mathcal{L}$, for which the existence of tractable algorithms is still open [18].

Thus in this paper we rely on the approximation of the deductive difference given in Definition 1 This approximation only requires comparing the classification hierarchies of $\mathcal{O}$ and $\mathcal{O}^{\prime}$ provided by an OWL 2 reasoner, and it has successfully been used in the past in the context of ontology integration [13].

Definition 1 (Approximation of the Deductive Difference). Let $A, B$ be atomic concepts from $\Sigma, \mathcal{O}$ and $\mathcal{O}^{\prime}$ be two OWL 2 ontologies, with $\Sigma$ a signature. We define the approximation of the $\Sigma$-deductive difference between $\mathcal{O}$ and $\mathcal{O}^{\prime}$ (denoted $\operatorname{diff} \approx \widetilde{\Sigma}\left(\mathcal{O}, \mathcal{O}^{\prime}\right)$ as the set of axioms of the form $A \sqsubseteq B$ satisfying: (i) $\mathcal{O} \not \models A \sqsubseteq$ $B$, and (ii) $\mathcal{O}^{\prime} \models A \sqsubseteq B$. 


\subsection{Violations and Mapping Repair}

As already discussed in Section 2.2, the notion of deductive difference can capture the novel entailments of an aligned ontology w.r.t. the input ontologies. However, some of these entailments may be undesired, and are called violations, stemming from erroneous mappings in $\mathcal{M}$, or from an inherent incompabilities between the input ontologies $\mathcal{O}_{1}$ and $\mathcal{O}_{2}$.

A set of mappings that leads to unsatisfiable classes in $\mathcal{O}_{1} \cup \mathcal{O}_{2} \cup \mathcal{M}$ is referred to as incoherent w.r.t. $\mathcal{O}_{1}$ and $\mathcal{O}_{2}$ [21], as formalized in Definition 2. Analogously, a set of mappings that leads to violations of the conservativity principle in the aligned ontology $\mathcal{O}_{1} \cup \mathcal{O}_{2} \cup \mathcal{M}$ is referred to as nonconservative w.r.t. $\mathcal{O}_{1}$ and $\left.\mathcal{O}_{2}[1] 31\right]$, as formalized in Definition 3 .

Definition 2 (Mapping Incoherence). A set of mappings $\mathcal{M}$ is incoherent with respect to $\mathcal{O}_{1}$ and $\mathcal{O}_{2}$, if a class $A$ exists in the signature of $\mathcal{O}_{1} \cup \mathcal{O}_{2}$ such that $\mathcal{O}_{1} \cup \mathcal{O}_{2} \not \mid A \equiv \perp$ and $\mathcal{O}_{1} \cup \mathcal{O}_{2} \cup \mathcal{M} \models A \sqsubseteq \perp$.

Definition 3 (Mapping Nonconservativity). A set of mappings $\mathcal{M}$ is nonconservative with respect to $\mathcal{O}_{1}$ and $\mathcal{O}_{2}$, if a pair of classes $A, B$ exist in the signature of $\mathcal{O}_{i}$, with $B \neq \perp$, such that $\mathcal{O}_{i} \forall A \sqsubseteq B$, and $\mathcal{O}_{1} \cup \mathcal{O}_{2} \cup \mathcal{M}=A \sqsubseteq B$.

More generally, an alignment being incoherent and/or nonconservative, is called problematic, as introduced in Definition 4

Definition 4 (Problematic Mappings). A set of mappings $\mathcal{M}$ between two ontologies $\mathcal{O}_{1}$ and $\mathcal{O}_{2}$ is problematic, if $\mathcal{M}$ is incoherent and/or nonconservative with respect to $\mathcal{O}_{1}$ and $\mathcal{O}_{2}$.

A problematic set of mappings $\mathcal{M}$ can be fixed by removing mappings from $\mathcal{M}$. This process is referred to as mapping repair (or repair for short).

Definition 5 (Mapping Repair). Let $\mathcal{M}$ be a problematic set of mappings w.r.t. $\mathcal{O}_{1}$ and $\mathcal{O}_{2}$. A set of mappings $\mathcal{R} \subseteq \mathcal{M}$ is a mapping repair for $\mathcal{M}$ w.r.t. $\mathcal{O}_{1}$ and $\mathcal{O}_{2}$ if $\mathcal{M} \backslash \mathcal{R}$ is not problematic w.r.t. $\mathcal{O}_{1}$ and $\mathcal{O}_{2}$.

A trivial repair is $\mathcal{R}=\mathcal{M}$, since an empty set of mappings is obviously nonproblematic. Nevertheless, the objective is to minimize a loss function over the alignment (e.g., to remove as few mappings as possible or to minimize the total confidence of the removed mappings). Minimal (mapping) repairs are typically referred to in the literature as mapping diagnosis [20] - a term coined by Reiter [25] and introduced to the field of ontology debugging in [28].
Definition 6 (Mapping diagnosis). Let $\mathcal{R}$ be a repair for $\mathcal{M}$ with respect to $\mathcal{O}_{1}$ and $\mathcal{O}_{2} . \mathcal{R}$ is a diagnosis if each $\mathcal{R}^{\prime} \subset \mathcal{R}$ is not a repair for $\mathcal{M}$ with respect to $\mathcal{O}_{1}$ and $\mathcal{O}_{2}$.

In the literature there are different approaches to compute a repair or diagnosis for an incoherent set of mappings. Early approaches were based on Distributed Description Logics (DDL) (e.g., [22 23|24]). Alternatively, if mappings are represented as OWL 2 axioms, a repair or diagnosis can also be computed using the state-of-the-art approaches for debugging and repairing OWL 2 ontologies, which rely on the extraction of justifications for the undesired entailments (e.g., [13|15|27|34]).

"A justification for an entailment in an ontology is a minimal subset of the ontology that is sufficient for the entailment to hold. The set of axioms corresponding to the justification is minimal in the sense that if an axiom is removed from the set, the remaining axioms no longer support the entailment." [10] Definition 7 formally introduces the notion of justification.

Definition 7 (Justification [10]). Given an ontology $\mathcal{O}$, and an entailment $\eta$ such that $\mathcal{O}=\eta, \mathcal{J}$ is a justification in $\mathcal{O}$ of $\eta$ if $\mathcal{J} \subseteq \mathcal{O}, \mathcal{J} \models \eta$, and for all $\mathcal{J}^{\prime} \subsetneq \mathcal{J}$ it is the case that $\mathcal{J}^{\prime} \not \neq \eta$.

In ontology matching scenarios the use of incomplete reasoning techniques to enhance scalability is very frequent (e.g., [1 $111|20| 26 \mid 31])$. Incomplete reasoning leads to an approximate repair $\mathcal{R} \approx$, i.e., there is no guaranteee that $\mathcal{M} \backslash \mathcal{R} \approx$ is nonproblematic, but the number of violations caused by the original set of mappings $\mathcal{M}$ tends to be reduced while minimizing the loss function over the original alignment.

Given that the justifications for an entailment are usually exponential in the size of the ontology, [10] approximate mapping repair techniques, based on the extraction of a single justification, has been successfully used in the past to achieve scalability (e.g., LogMapFull [11]). For this reason, our empirical evaluation does not only consider the (limited) extraction of all the justifications, but also the computation of a single one, as described in details in [10].

\section{Experimental Evaluation}

This section describes the conducted experimental evaluation. In Section 3.1 we present the used datasets and mapping sets. Section 3.2 introduces the evaluation setting. The obtained results are discussed in Section 3.3 


\subsection{Datasets}

The datasets are based on the OAEI, an international campaign for the systematic evaluation of ontology matching systems. The matching problems in the OAEI are organised in several tracks, with each track involving different kinds of test ontologies [368]. In this paper, we have focused on the anatomy, largebio, library and conference tracks. For largebio we used both the small setting, in which reduced fragments of FMA, NCI and SNOMED CT are employed (where the fragments are relevant portions of one of the ontologies with respect to the other two), and the big setting, employing the whole ontologies (at the exception of SNOMED CT, for which a large fragment for both FMA and NCI is used). Library is composed by not very expressive medium-sized ontologies, while conference ontologies are very expressive but of limited size. Anatomy is composed by a fragment of NCI ontology (named HUMAN in this context to avoid confusion with the largebio dataset) involving human anatomy, that should be matched with an ontology describing the anatomy of mice (called MOUSE ontology). Table 1 summarizes the metrics of the selected ontology pairs for the evaluation, while Tables 2,4 provides the details about the selected subset of mapping sets computed by ontology matching systems participating in the OAEI 2013 and 2014 campaigns. Due to the excessive time required for running a so expensive evaluation, we were forced to select only a representative subset of the computed mappings sets (we have selected, for each track, the alignments with the highest or lowest precision and recall values) 1 Please refer to [36] for more information about the datasets and ontology matching systems.

\subsection{Evaluation Settings}

System Details. The test environment consists of a desktop computer equipped with $32 G B$ DDR3 RAM at $1333 \mathrm{MHz}$ and an $A M D$ Fusion $F X 4350$ (quadcore, each running at $4.2 \mathrm{GHz}$ ) as $C P U$. The dataset is stored on a $128 G B S S D$, where the operating system (Ubuntu 12.04, 64-bit version) is installed. The employed build of Java Runtime Environment (JRE) is 1.8.0_45-b14, while the one for the Oracle 64-Bit

\footnotetext{
${ }^{1}$ Due to space reasons we can only present a subset of the computed evaluation, a technical report with the full analysis is available at ftp://ftp.disi.unige.it/person/ SolimandoA/aijournaltr.pdf
}

Table 2

Metrics about the relevant mapping sets of the largebio dataset.

\begin{tabular}{|c|c|c|c|}
\hline Ontology 1 & Ontology 2 & \# Mappings & Matching System \\
\hline FMA & $\mathrm{NCI}$ & 5862 & $\mathrm{AML}_{14}$ (BIG) \\
\hline FMA & NCI & 5686 & GOMMA $_{13}(\mathrm{BIG})$ \\
\hline FMA & $\mathrm{NCI}$ & 3788 & IAMA $_{13}(\mathrm{BIG})$ \\
\hline FMA & $\mathrm{NCI}$ & 6823 & $\operatorname{LogMapBio}_{14}$ (BIG) \\
\hline FMA & NCI & 2806 & OMReasoner $_{14}(\mathrm{BIG})$ \\
\hline FMA & NCI & 6048 & Reference $_{13}(\mathrm{BIG})$ \\
\hline FMA & $\mathrm{NCI}$ & 5518 & $\mathrm{YAM}++_{13}(\mathrm{BIG})$ \\
\hline FMA & SNOMED & 12384 & $\mathrm{AML}_{14}(\mathrm{BIG})$ \\
\hline FMA & SNOMED & 11294 & $\mathrm{GOMMA}_{13}(\mathrm{BIG})$ \\
\hline FMA & SNOMED & 3198 & IAMA $_{13}(\mathrm{BIG})$ \\
\hline FMA & SNOMED & 13704 & $\operatorname{LogMapBio}_{14}(\mathrm{BIG})$ \\
\hline FMA & SNOMED & 18016 & Reference $_{13}$ (BIG) \\
\hline FMA & SNOMED & 13684 & YAM++13 $(\mathrm{BIG})$ \\
\hline SNOMED & NCI & 25252 & $\mathrm{AML}_{14}(\mathrm{BIG})$ \\
\hline SNOMED & $\mathrm{NCI}$ & 24880 & $\mathrm{GOMMA}_{13}(\mathrm{BIG})$ \\
\hline SNOMED & NCI & 17686 & IAMA $_{13}(\mathrm{BIG})$ \\
\hline SNOMED & $\mathrm{NCI}$ & 24984 & $\operatorname{LogMapBio}_{14}(\mathrm{BIG})$ \\
\hline SNOMED & $\mathrm{NCI}$ & 37688 & Reference $_{13}(\mathrm{BIG})$ \\
\hline SNOMED & NCI & 25200 & $\mathrm{YAM}++_{13}(\mathrm{BIG})$ \\
\hline FMA & $\mathrm{NCI}$ & 5380 & $\mathrm{AML}_{14}$ \\
\hline FMA & NCI & 5252 & GOMMA $_{13}$ \\
\hline FMA & $\mathrm{NCI}$ & 3502 & IAMA $_{13}$ \\
\hline FMA & $\mathrm{NCI}$ & 5960 & MaasMatch $_{14}$ \\
\hline FMA & $\mathrm{NCI}$ & 5781 & $\operatorname{LogMapBio}_{14}$ \\
\hline FMA & $\mathrm{NCI}$ & 2724 & OMReasoner $_{14}$ \\
\hline FMA & NCI & 5122 & $\mathrm{YAM}++_{13}$ \\
\hline FMA & SNOMED & 13582 & $\mathrm{AML}_{14}$ \\
\hline FMA & SNOMED & 7332 & GOMMA $_{13}$ \\
\hline FMA & SNOMED & 2500 & IAMA $_{13}$ \\
\hline FMA & SNOMED & 12884 & $\operatorname{LogMapBio}_{14}$ \\
\hline FMA & SNOMED & 16232 & MaasMatch $_{14}$ \\
\hline FMA & SNOMED & 3040 & OMReasoner $_{14}$ \\
\hline FMA & SNOMED & 13270 & YAM++ $_{13}$ \\
\hline SNOMED & $\mathrm{NCI}$ & 28262 & $\mathrm{AML}_{14}$ \\
\hline SNOMED & $\mathrm{NCI}$ & 21110 & GOMMA $_{13}$ \\
\hline SNOMED & $\mathrm{NCI}$ & 16812 & IAMA $_{13}$ \\
\hline SNOMED & $\mathrm{NCI}$ & 28711 & $\operatorname{LogMapBio}_{14}$ \\
\hline SNOMED & $\mathrm{NCI}$ & 14240 & OMReasoner $_{14}$ \\
\hline SNOMED & NCI & 23344 & YAM++13 \\
\hline
\end{tabular}

Java Virtual Machine (JVM) is the 25.45-b02 (mixed mode). The amount of memory allocated for the heap of the JVM is $12 \mathrm{~GB}$, the processes not involved in the evaluation require approximately $3 \mathrm{~GB}$ of space, thus leaving $17 \mathrm{~GB}$ of free RAM (plus $1.8 \mathrm{~GB}$ of swap memory, that is not used unless totally necessary ${ }^{2}$.

Tested Reasoners. The versions of the employed reasoners are: (i) Konclude 0.6.0-408 64-bit [33] (linux

\footnotetext{
${ }^{2}$ This behaviour is enforced by means of the swappiness Linux kernel parameter set to 0 , see http://en.wikipedia.org/ wiki/Swappiness for more information.
} 
Table 1

Metrics about the ontologies employed in the evaluation.

\begin{tabular}{|c|c|c|c|c|c|}
\hline Ontology & Track & \#Concepts & \#DatatypeP. & \#ObjectP. & DL \\
\hline MOUSE & Anatomy & 2744 & 0 & 3 & $\mathcal{A L \mathcal { E } ( D )}$ \\
\hline HUMAN & Anatomy & 3304 & 0 & 2 & $\mathcal{S}(\boldsymbol{D})$ \\
\hline CMT & Conference & 36 & 10 & 49 & $\mathcal{A L C \mathcal { I N } ( \boldsymbol { D } )}$ \\
\hline CONFERENCE & Conference & 60 & 18 & 46 & $\mathcal{A} \mathcal{L C \mathcal { H } \mathcal { I }}(\boldsymbol{D})$ \\
\hline CONFOF & Conference & 38 & 23 & 13 & $\mathcal{S I \mathcal { N }}(\boldsymbol{D})$ \\
\hline EKAW & Conference & 74 & 0 & 33 & $\mathcal{S H \mathcal { I N }}$ \\
\hline IASTED & Conference & 140 & 3 & 38 & $\mathcal{A L C \mathcal { I N } ( D )}$ \\
\hline SIGKDD & Conference & 49 & 11 & 17 & 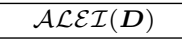 \\
\hline FMA (NCI) & LargebioSmall & 3696 & 24 & 0 & $\mathcal{A L C \mathcal { N }}(\boldsymbol{D})$ \\
\hline FMA (SNOMED) & LargebioSmall & 10157 & 24 & 0 & $\mathcal{A L C \mathcal { N }}(\boldsymbol{D})$ \\
\hline NCI (FMA) & LargebioSmall & 6488 & 0 & 63 & $\mathcal{A L C}$ \\
\hline NCI (SNOMED) & LargebioSmall & 23958 & 0 & 82 & $\mathcal{A L C H}$ \\
\hline SNOMED (FMA) & LargebioSmall & 13412 & 0 & 18 & $\mathcal{A L \mathcal { E } R}$ \\
\hline SNOMED (NCI) & LargebioSmall & 51128 & 0 & 51 & $\mathcal{A L \mathcal { E } R}$ \\
\hline FMA & LargebioBig & 78988 & 54 & 0 & $\mathcal{A L C \mathcal { N }}(\boldsymbol{D})$ \\
\hline NCI & LargebioBig & 66724 & 1 & 123 & $\mathcal{A L C \mathcal { H } ( \boldsymbol { D } )}$ \\
\hline SNOMED (NCI and FMA) & LargebioBig & 122464 & 1 & 55 & $\mathcal{A L \mathcal { E } R}$ \\
\hline STW & Library & 6575 & 0 & 0 & $\mathcal{A L}$ \\
\hline TheSoz & Library & 8376 & 0 & 0 & $\mathcal{A L}$ \\
\hline
\end{tabular}

Table 3

Metrics about the relevant mapping sets of the conference dataset.

\begin{tabular}{|c|c|c|c|}
\hline Ontology 1 & Ontology 2 & \# Mappings & Matching System \\
\hline \hline CMT & IASTED & 10 & AML $_{14}$ \\
\hline CMT & IASTED & 10 & AMLbk $_{13}$ \\
\hline CMT & IASTED & 32 & MaasMatch $_{14}$ \\
\hline CMT & IASTED & 8 & Reference $_{14}$ \\
\hline CMT & IASTED & 15 & XMapGen $_{13}$ \\
\hline CMT & IASTED & 11 & XMapSig $_{13}$ \\
\hline CONFOF & IASTED & 10 & AML $_{14}$ \\
\hline CONFOF & IASTED & 10 & AMLbk $_{13}$ \\
\hline CONFOF & IASTED & 18 & Reference $_{14}$ \\
\hline CONFOF & IASTED & 19 & XMapGen $_{13}$ \\
\hline CONFOF & IASTED & 9 & XMapSig $_{13}$ \\
\hline CONFOF & IASTED & 16 & YAM++ $_{13}$ \\
\hline CONFERENCE & EKAW & 164 & MaasMatch $_{14}$ \\
\hline CONFERENCE & IASTED & 68 & MaasMatch $_{14}$ \\
\hline CONFERENCE & IASTED & 12 & AML $_{14}$ \\
\hline CONFERENCE & IASTED & 12 & AMLbk $_{13}$ \\
\hline CONFERENCE & IASTED & 28 & Reference $_{14}$ \\
\hline CONFERENCE & IASTED & 17 & XMapGen $_{13}$ \\
\hline CONFERENCE & IASTED & 13 & XMapSig $_{13}$ \\
\hline CONFERENCE & IASTED & 12 & YAM++ $_{13}$ \\
\hline IASTED & SIGKDD & 70 & AOTL $_{14}$ \\
\hline IASTED & SIGKDD & 68 & MaasMatch $_{14}$ \\
\hline
\end{tabular}

binaries), (ii) ELK 0.4.2 [17] (iii) Pellet 2.3.1 [30], (iv) HermiT 1.3.8 [9].

ELK, Pellet, and HermiT implement the OWLReasoner interface of the $O W L-A P I$ and they all are called

\footnotetext{
${ }^{3}$ Version compiled on the 29th of May 2015 from the sources available at https://github.com/klinovp/elk/tree/ feature/tracing-complete
}

Table 4

Metrics about the relevant mapping sets of the anatomy and library datasets.

\begin{tabular}{|c|c|c|c|}
\hline Ontology 1 & Ontology 2 & \# Mappings & Matching System \\
\hline \hline MOUSE & HUMAN & 2956 & AML $_{14}$ \\
\hline MOUSE & HUMAN & 2954 & AMLbk $_{13}$ \\
\hline MOUSE & HUMAN & 5400 & AOT $_{14}$ \\
\hline MOUSE & HUMAN & 336 & AOTL $_{14}$ \\
\hline MOUSE & HUMAN & 3077 & GOMMAbk $_{13}$ \\
\hline MOUSE & HUMAN & 1962 & IAMA $_{13}$ \\
\hline MOUSE & HUMAN & 3107 & LogMapBio $_{14}$ \\
\hline MOUSE & HUMAN & 2235 & LogMapC $_{14}$ \\
\hline MOUSE & HUMAN & 4024 & MaasMatch $_{13}$ \\
\hline MOUSE & HUMAN & 2206 & ODGOMS $_{13}$ \\
\hline MOUSE & HUMAN & 3032 & Reference $_{14}$ \\
\hline MOUSE & HUMAN & 1891 & RSDLWB $_{14}$ \\
\hline MOUSE & HUMAN & 1872 & WeSeE $_{13}$ \\
\hline MOUSE & HUMAN & 2056 & WMatch $_{13}$ \\
\hline MOUSE & HUMAN & 2790 & YAM++ $_{13}$ \\
\hline STW & TheSoz & 9582 & AML $_{13}$ \\
\hline STW & TheSoz & 7254 & AML $_{14}$ \\
\hline STW & TheSoz & 12032 & Hertuda $_{13}$ \\
\hline STW & TheSoz & 378 & IAMA $_{13}$ \\
\hline STW & TheSoz & 5684 & LogMap $_{13}$ \\
\hline STW & TheSoz & 2925 & LogMapC $_{14}$ \\
\hline STW & TheSoz & 8922 & MaasMatch $_{14}$ \\
\hline STW & TheSoz & 7794 & ODGOMS $_{13}$ \\
\hline STW & TheSoz & 6322 & Reference $_{14}$ \\
\hline STW & TheSoz & 1624 & StringsAuto $_{13}$ \\
\hline STW & TheSoz & 342 & RSDLWB $_{14}$ \\
\hline STW & TheSoz & 11948 & Xmap $_{14}$ \\
\hline STW & TheSoz & 80686 & XMapGen $_{13}$ \\
\hline STW & TheSoz & 2870 & XMapSig $_{13}$ \\
\hline STW & TheSoz & 7940 & YAM++ $_{13}$ \\
\hline
\end{tabular}


on a fresh thread. A timeout on the classification task is enforced by killing the thread after reaching the timeout value, times are measured using the getNanoSec function, because it measures the elapsed time without skew corrections 4

$E L K$ is a (very fast) reasoner for the OWL 2 EL profile, thus it cannot guarantee complete results for ontologies outside this profile.

Konclude does not implement the $O W L$-API's $O W L$ Reasoner interface and its invocation through OWLlink 1.2.1 is raising an OWLlinkReasonerRuntimeException exception caused by an IndexOutOfBoundsException exception during the parsing of most of the ontologies in our dataset (expressed in OWL/XML format). Thus, Konclude is instead called using an external process ${ }^{5}$ using the ProcessBuilder class. ${ }^{6}$ and it is allowed to use all the available cores. For Konclude, timeout on classification is enforced using timeout program for Linux $\sqrt{7}$ and wall-clock time is measured using the time program 8

Note that due to its invocation, the measured time for Konclude also includes the loading time of the aligned ontology. However, given that we aim at verifying the feasibility of using full OWL 2 reasoners in a mapping diagnosis context, and not at comparing reasoners, all these biases are not influencing our analysis.

It was not possible to extend our analysis to $\mathrm{FaCT++}$ because its invocation using $J N I$ is permanently failing with a StackOverflowError.

Justification Extractor. Our evaluation is based on the black-box justification extractor described in [10] 9 Black-box extractors typically allow to use any reasoner implementing the axiom pinpointing service. In addition to our previous evaluation, we have also compared the performance of the aforementioned blackbox approach using ELK 0.4.2, and the glass-box trace

4 https://docs.oracle.com/javase/8/docs/api/ java/lang/System.html\#nanoTime--

${ }^{5}$ Konclude is runned with "Konclude classification -w AUTO -i aligneOntology.owl"

thttps://docs.oracle.com/javase/8/docs/api/ java/lang/ProcessBuilder.html

${ }^{7}$ With the command "timeout - preserve-status -s TERM timeoutVal cmd"

${ }^{8}$ Using "/usr/bin/time -f \%E cmd" command.

${ }^{9}$ Current version available at https://github.com/ matthewhorridge/owlexplanation For the experiments we used the version available here: https://github.com/ protegeproject/mvn-repo/tree/master/releases/ org/semanticweb/owl/explanation/3.3.0

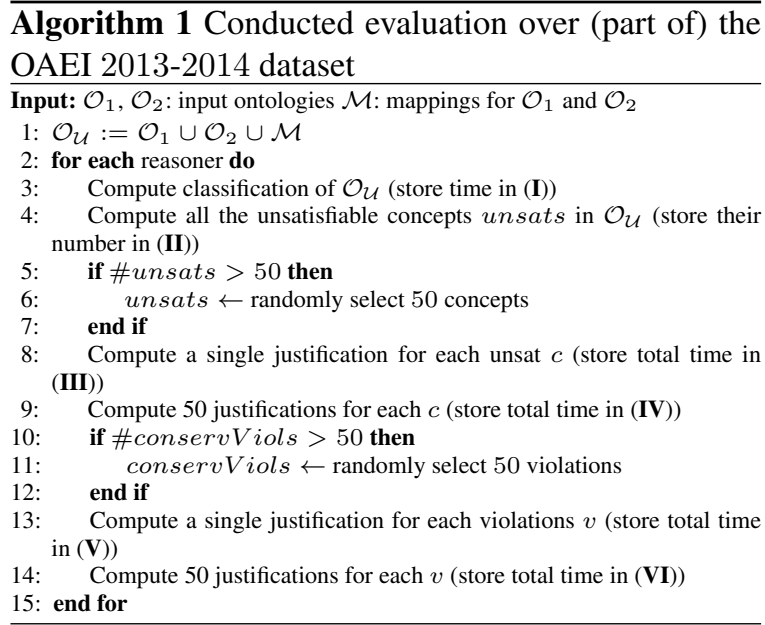

extraction technique offered by $E L K$ reasoner [16]. The tracing functionality offerred by $E L K$ keeps track of the subset of the ontology used for the entailment check of the axiom of interest, then the black-box justification module is run only against this relevant subset of the ontology, allowing, in principle, a significant reduction of the required runtime. [10] When we refer to this additional functionality offered by $E L K$, the reasoner will be referred to as $E L K_{\text {trace }}$.

\subsection{Conducted Evaluation}

The evaluation algorithm is presented in Algorithm 1. which takes as input a pair of ontologies $\left(\mathcal{O}_{1}\right.$ and $\mathcal{O}_{2}$ ) and an alignment $\mathcal{M}$ between them from the datasets described in Section 3.1. For each of the available reasoners we compute the classification ${ }^{10}$ and record the classification times in seconds (see Tables 5 . 9 and Class.(s) in Tables 10 31). Then, if the classification succeeds, we record the number of unsatisfiable concepts (\#Unsat in Tables 10 31). For at most 50 of them, we compute justification 11 (a single one and up to a maximum of 50 justifications, recording the total time in seconds required for completing the respective operations (1Just.(s) and 50Just.(s) in Tables 10 31 respectively). In addition, we also keep track of the percentage of violations for which neither error nor timeout occurred (\%1JustOK and \%50JustOK). When

\footnotetext{
${ }^{10}$ With a timeout of $10,60,20$ and 10 minutes for anatomy, largebio, library and conference, respectively.

${ }^{11}$ With a timeout of 60 seconds to find each new justification. $E L K_{\text {trace }}$ has a timeout of 60 seconds for computing the trace, and than another timeout of 60 seconds for computing each justification.
} 
a timeout or an error occurs, the measured time is only a lower-bound of the real required time.

Analogously, the number of detected conservativity violations are recorded (\#Viols in Tables 10 31, and, for at most 50 of them, we compute justifications.

Notice that when computing more than one justification, the timeout is enforced for each single justification computation, and reaching a timeout on one of them stops the whole computation. This is motivated by the fact that the search space for justifications is explored by increasing the number of axioms composing them, and it is therefore reasonable that at the first failure the justification module will not be able to find other ones within the given time limit.

Classification. In Tables 5,9 the classification time for a selection of the testcases is shown.

For the anatomy dataset (Table 5, all the reasoners succesfully managed to compute the classification of the aligned ontology. The only reasoner that experienced problems was Pellet, that permanently raised a ConcurrentModificationException exception on several aligned ontologies of this dataset.

In the largebio-small dataset (Table 6), for the mapping sets involving FMA and NCI, none of the reasoners failed at classifying the aligned ontology. For the FMA and SNOMED testcase, Pellet failed to classify, due to timeouts (T/OUT), half of the cases. For the integration of SNOMED and NCI, Pellet failed due to timeouts in all the cases, and exclusively ELK could classify the aligned ontology in 3 out of 7 testcases ${ }^{12}$ Konclude, for instance, failed with an out of memory error (OOM), and HermiT reached the timeout.

For the largebio-big dataset (Table 7), not all the mapping sets are available because some of the ontology matchers failed at computing the alignment for this extended case. Regarding classification, the results are very similar to the small version of the dataset, with a little increase of the number of timeouts for Pellet due to the increase of size of the aligned ontologies.

For library (Table 8), instead, the reasoners succeeded in most of the cases (with the partial exception of Pellet), but only Konclude managed to classify, within the timeout, the integrated ontology via the mappings computed by XMapGen. These mappings include an extremely high number of many to many

\footnotetext{
${ }^{12}$ Note that $E L K$ is an OWL 2 EL reasoner and since NCI falls outside the OWL 2 EL profile, the classification computed by ELK for the integration of SNOMED and NCI is incomplete.
}

correspondences, that caused problems to all the reasoners but Konclude.

Concerning conference, the classification could be performed in the vast majority of the cases, with only a single failure for both HermiT and Pellet. In Table 9 we only report the cases in which the classification either required a time greater than 1 second, or during which a timeout or error occurred.

Computation of Justifications for Unsatisfiabilities. Tables 10 30. instead, show the details for justification computation for the existing unsatisfiabilites, for relevant cases. For the library dataset, the results are omitted due to the lack of unsatisfiable classes in the aligned ontologies (the input ontologies are simple and they do not contain disjointness axioms). Note that Konclude, since it was invoked from the command line, could not be evaluated on the justification extraction tasks, due to the limitation of using it from the command line. Missing rows mean that the corresponding reasoner failed at classifying the integrated ontology, due to timeout or an error, and has been excluded.

Note that, the computed times in the Tables 10 30 are only for 50 unsatisfiable classes. Thus, the total times given below for all unsatisfiable classes have been extrapolated from these results.

A subset of the results for the anatomy dataset is reported in Tables 10 11. Consider for instance Table 10a. which presents the justification extraction for the mapping set computed by MaasMatch. Computing a single justification for each of the 5,972 unsatisfiable classes, would require for $E L K 2 \mathrm{~h}$ ( 68 s for 50 unsatisfiable classes), while $>11$ days for computing fifty of them ( $>2 \mathrm{~h}$ for 50 unsatisfiable classes).

The available version of ELK $K_{\text {trace }}$ is still in beta quality, and therefore we experienced some problems in the justification extraction process. For this reason, despite the great improvement in the runtime for computing justifications, it exhibited a high number of errors during the evaluation. For instance, for the present task, $E L K_{\text {trace }}$ did not manage to correctly compute any justification. When HermiT is used, $>37 \mathrm{~m}$ and $>90 \mathrm{~h}$ would be required, respectively. These times are surprisingly lower than the corresponding for $E L K$, despite the latter is an approximated reasoner.

While already the reported times could be considered to be incompatible with "online" repair scenarios, we also need to consider that the runtime is a lowerbound, limited to at most 50 justifications, of the one 
extracting all the justifications, whose number is usually exponential in the size of the ontology.

Considering the conference dataset, composed by small sized ontologies having high expressivity, we also find cases that could not be compatible with an "online" mapping repair (e.g., almost two hours for HermiT in Table 14a $>53 \mathrm{~m}$ for Pellet in Table 13a and $>47 \mathrm{~m}$ for $E L K$ in Table $15 \mathrm{a}$ ). Notice also that in this last case, only $16 \%$ of the computations involving Pellet finished before the timeout, and therefore the total runtime is expected to be much higher. The worst result for $E L K_{\text {trace }}$, instead, is in Table 15 a, with a runtime $>22 \mathrm{~m}$.

For the largebio datasets, shown in Tables $16,30$. both big and small variants, the values are definitely higher and not affordable for an online repair process. Computing a single justification for each unsatisfiable concept in the largebio testcase of Table 20a would require $>63 \mathrm{~h}$ for $E L K,>6 \mathrm{~h}$ for $E L K_{\text {trace }},>68 \mathrm{~h}$ for Her$m i T$, while $>613$ days, $>485$ days and $>51$ days for computing 50 of them, respectively.

Even considering a testcase of largebio-small, with a fairly limited number of unsatisfiable classes (2058 for the mapping set involving FMA and SNOMED and computed by GOMMA, Table 25a, the runtime is still prohibitive if more than one justification is computed. Indeed, computing a single justification would require $>10 \mathrm{~m}$ for $E L K,>44 \mathrm{~s}$ for $E L K_{\text {trace }},>6 \mathrm{~m}$ for HermiT and $>4 \mathrm{~m}$ for Pellet, while $>24$ days, $>32 \mathrm{~h},>10$ days and $>7$ days for computing 50 of them, respectively.

It is evident that the proposed runtimes for largebio might be only acceptable in an off-line mapping repair process for the small testcases, while they are not affordable for the largest ones.

Computation of Justifications for Conservativity Violations. In Tables 10 31 we show the details for justification computation for the conservativity violations, restricted to the relevant cases.

What is evident from the analyzed testcases is that, the average runtime required for computing the justifications for a conservativity violation (that is, a subsumption axiom between named classes) is in general lower than the time required for computing the justifications for an unsatisfiable class.

On the other hand, it is also true that the number of violations is usually higher than the number of unsatisfiabilities, with millions of violations like in the $l i$ brary testcase, and this is balancing the required time for computing justifications.
For instance, for the anatomy dataset, in Table $10 \mathrm{~b}$ computing a single justification requires $>25 \mathrm{~m}$ for $E L K,>5 \mathrm{~h}$ for $E L K_{\text {trace }}$, and $>4 \mathrm{~m}$ for HermiT, while $>50 \mathrm{~h},>43 \mathrm{~h}$, and $>16 \mathrm{~h}$ for computing 50 justifications, respectively. It is again notable that the required runtimes for HermiT are lower than the corresponding for $E L K$ (both variants).

The conference dataset exhibits a very limited number of conservativity violations, in general, favored by the extremely reduced size of the signature of the involved ontologies. An exception is represented by the testcase of Table $13 \mathrm{~b}$, where computing a single justification requires less than 2 seconds for all the tested reasoners, but computing 50 justifications caused $32 \%$ of timeouts for Pellet. All the other reasoners were still able to accomplish the task within one minute.

For largebio, Table 26b presents a very limited number of conservativity violations (2270), that paired with the reduced runtime for the computation of their justifications, as already discussed, requires at most $10 \mathrm{sec}$ onds for computing a single justification, and at most 39 minutes for computing 50 justifications. Even if the required time can appear to be limited, we need to remind again that in order to provide a complete and minimal repair, all the (possibly exponentially many) justifications must be computed, while the proposed runtime is for computing at most 50 justifications.

In other largebio testcases, such as that described in Table 28b, ELK would need 12 hours to compute a single justification ( $E L K_{\text {trace }}$ raised an error on $90 \%$ of the computations and its runtime cannot be compared), while $>127$ days would be required by $E L K$, and $>5$ days by $E L K_{\text {trace }}$ for computing 50 justifications.

The highest number of conservativity violations occurs in the library dataset, favored by the extremely high number of many to many correspondences that its mapping sets exhibit. For instance, in the testcase of Table $31 \mathrm{~b}$ the required time for the computation of a single justification is $>229$ days for $E L K$, and $>18$ days for HermiT, while for computing 50 justifications the runtime is $>48$ and $>7$ years, respectively. The results for $E L K_{\text {trace }}$ are omitted because, for the selected testcases, all the single justification computation failed, and all that for multiple justifications reached the timeout. Similarly to the anatomy testcase, also for library the runtime for justification computation with HermiT has been lower than that of ELK.

It is again evident that despite the smaller runtime required for computing justifications for conservativity violations, the total runtime would be prohibitive in the majority of the cases, also for off-line repair scenarios. 


\section{Conclusions}

In this paper, we have extended previous evaluations of the feasibility of using OWL 2 reasoning capabilities in mapping repair related tasks, under different aspects. Firstly, the evaluation has been extended to extract more justifications than in the previous evaluation, in order to provide more accurate results. Additionally, the black-box justification extraction method has also been compared against glass-box techniques offered by $E L K$ reasoner, which provides the capability of extracting a trace of the relevant subset of an ontology, for the entailment check of a given axiom. Finally, we also analyzed the performances of justification extraction for repairing the so-called conservativity principle.

Our empirical results on the performances of several top-level reasoners suggest that the classification of the integration of medium/large size ontologies via mappings is hard to compute for current OWL 2 reasoners. Furthermore, when OWL 2 reasoners are to be used in mapping repair tasks, the computation time increases considerably, and in the majority of the cases it becomes impractical, even when restricting to reasoners for one of the OWL 2 profiles. From the extended empirical evaluation presented in this paper, the problem is even exacerbated when considering conservativity violations, in addition to consistency ones.

Hence, the integration of ontologies via mappings seems an ideal reasoning benchmarks, and its hardness motivates the interest in approximated repair techniques in the context of ontology matching.

\section{Acknowledgements}

This work was supported by the EU FP7 IP project Optique (no. 318338) and the EPSRC project Score!. Alessandro Solimando has been fully supported by the AI*IA 2014 Mobility Grant for visiting Ernesto Jiménez-Ruiz at the Knowledge Representation and Reasoning group, University of Oxford, UK. We also thank the unvaluable help provided by Bernardo Cuenca Grau and Ian Horrocks.

\section{References}

[1] Alessandro Solimando, Ernesto Jiménez-Ruiz and Giovanna Guerrini. A Multi-strategy Approach for Detecting and Correcting Conservativity Principle Violations in Ontology Alignments. In OWLED Workshop, volume CEUR-WS 1265, pages 13-24, 2014.
[2] Alexander Borgida and Luciano Serafini. Distributed Description Logics: Assimilating Information from Peer Sources. $J$. Data Sem., 1:153-184, 2003.

[3] Bernardo Cuenca Grau, Zlatan Dragisic, Kai Eckert, et al. Results of the Ontology Alignment Evaluation Initiative 2013. In Ontology Matching (OM), 2013.

[4] Bernardo Cuenca Grau, Ian Horrocks, Boris Motik, Bijan Parsia, Peter F. Patel-Schneider, and Ulrike Sattler. OWL 2: The next step for OWL. J. Web Sem., 6(4):309-322, 2008.

[5] Jérôme David, Jérôme Euzenat, François Scharffe, and Cássia Trojahn. The Alignment API 4.0. J. Sem. Web, 2(1):3-10, 2011.

[6] Zlatan Dragisic, Kai Eckert, Jérôme Euzenat, Daniel Faria, et al. Results of the ontology alignment evaluation initiative 2014. In Int'l Workshop on Ontology Matching (OM), pages 61-104, 2014

[7] Jérôme Euzenat. Semantic Precision and Recall for Ontology Alignment Evaluation. In Int'l Joint Conf. on Artif. Intell. (IJCAI), pages 348-353, 2007.

[8] Jérôme Euzenat, Christian Meilicke, Heiner Stuckenschmidt, Pavel Shvaiko, and Cássia Trojahn. Ontology Alignment Evaluation Initiative: Six Years of Experience. J. Data Sem., 15:158-192, 2011.

[9] Birte Glimm, Ian Horrocks, Boris Motik, Giorgos Stoilos, and Zhe Wang. Hermit: An OWL 2 reasoner. J. Autom. Reasoning, 53(3):245-269, 2014.

[10] Matthew Horridge. Justification Based Explanation in Ontologies. Ph.D. thesis, School. Ph.D thesis, School of Computer Science, The University of Manchester, 2011.

[11] Ernesto Jiménez-Ruiz and Bernardo Cuenca Grau. LogMap: Logic-based and Scalable Ontology Matching. In Int'l Sem. Web Conf. (ISWC), pages 273-288, 2011.

[12] Ernesto Jiménez-Ruiz, Bernardo Cuenca Grau, and Ian Horrocks. On the feasibility of using OWL 2 DL reasoners for ontology matching problems. In OWL Reasoner Evaluation Workshop (ORE), 2012.

[13] Ernesto Jiménez-Ruiz, Bernardo Cuenca Grau, Ian Horrocks, and Rafael Berlanga. Ontology integration using mappings: Towards getting the right logical consequences. In Eur. Sem. Web Conf., 2009.

[14] Ernesto Jiménez-Ruiz, Christian Meilicke, Bernardo Cuenca Grau, and Ian Horrocks. Evaluating Mapping Repair Systems with Large Biomedical Ontologies. In Description Logics, pages 246-257, 2013.

[15] Aditya Kalyanpur, Bijan Parsia, Matthew Horridge, and Evren Sirin. Finding all justifications of OWL DL entailments. Int' $l$ Sem. Web Conf. (ISWC), pages 267-280, 2007.

[16] Yevgeny Kazakov and Pavel Klinov. Goal-directed tracing of inferences in EL ontologies. In 13th International Semantic Web Conference, pages 196-211, 2014.

[17] Yevgeny Kazakov, Markus Krötzsch, and Frantisek Simancik. Concurrent Classification of EL Ontologies. In Int'l Sem. Web Conf. (ISWC), pages 305-320, 2011.

[18] Boris Konev, Dirk Walther, and Frank Wolter. The Logical Difference Problem for Description Logic Terminologies. In Int'l Joint Conf. on Automated Reasoning (IJCAR), pages 259274, 2008.

[19] Roman Kontchakov, Frank Wolter, and Michael Zakharyaschev. Can you Tell the Difference Between DL-Lite Ontologies? In Int'l Conf. on Knowl. Representation and Reasoning (KR), 2008. 
[20] Christian Meilicke. Alignments Incoherency in Ontology Matching. PhD thesis, University of Mannheim, 2011.

[21] Christian Meilicke and Heiner Stuckenschmidt. Incoherence as a basis for measuring the quality of ontology mappings. In Ontology Matching (OM), 2008.

[22] Christian Meilicke, Heiner Stuckenschmidt, and Andrei Tamilin. Repairing ontology mappings. In Proc. of AAAI Conf. on Artif. Intell., pages 1408-1413, 2007.

[23] Christian Meilicke, Heiner Stuckenschmidt, and Andrei Tamilin. Reasoning Support for Mapping Revision. J. Log. Comput., 19(5), 2009.

[24] Jyotishman Pathak and Christopher G. Chute. Debugging Mappings between Biomedical Ontologies: Preliminary Results from the NCBO BioPortal Mapping Repository. In Int'l Conf. on Biomedical Ontology (ICBO), 2009.

[25] Raymond Reiter. A Theory of Diagnosis from First Principles. Artif. Intell., 32(1), 1987.

[26] Emanuel Santos, Daniel Faria, Cátia Pesquita, and Francisco Couto. Ontology Alignment Repair Through Modularization and Confidence-based Heuristics. arXiv:1307.5322 preprint, 2013.

[27] Stefan Schlobach. Debugging and Semantic Clarification by Pinpointing. In Eur. Sem. Web Conf. (ESWC), pages 226-240. Springer, 2005.

[28] Stefan Schlobach and Ronald Cornet. Non-standard Reasoning Services for the Debugging of Description Logic Terminologies. In Int'l Joint Conf. on Artif. Intell. (IJCAI), pages 355-362, 2003.

[29] Pavel Shvaiko and Jérôme Euzenat. Ontology Matching: State of the Art and Future Challenges. IEEE Transactions on Knowl. and Data Eng. (TKDE), 2012.

[30] Evren Sirin, Bijan Parsia, Bernardo Cuenca Grau, Aditya Kalyanpur, and Yarden Katz. Pellet: A practical OWL-DL reasoner. J. Web Sem., 5(2):51-53, 2007.

[31] Alessandro Solimando, Ernesto Jiménez-Ruiz, and Giovanna Guerrini. Detecting and correcting conservativity principle violations in ontology-to-ontology mappings. In Int'l Semantic Web Conference (ISWC), volume LNCS 8797, pages 1-16, 2014.

[32] Alessandro Solimando, Ernesto Jiménez-Ruiz, and Giovanna Guerrini. On the Feasibility of Using OWL 2 Reasoners in Ontology Alignment Repair Problems. In OWL Reasoner Evaluation Workshop (ORE), pages 60-67, 2015.

[33] Andreas Steigmiller, Thorsten Liebig, and Birte Glimm. Konclude: System description. J. Web Sem., 27:78-85, 2014.

[34] Boontawee Suntisrivaraporn, Guilin Qi, Qiu Ji, and Peter Haase. A Modularization-Based Approach to Finding All Justifications for OWL DL Entailments. In Asian Sem. Web Conf. $(A S W C), 2008$ 
Table 5

Classification times (s), anatomy dataset with selected mapping sets.

\begin{tabular}{|c|c|c|c|c|c|c|c|c|}
\hline & $\mathbf{A M L}_{14}$ & AMLBK $_{1}$ & $\mathbf{A O T}_{14}$ & AOTL $_{14}$ & GOMMABK $_{13}$ & IAMA $_{13}$ & LogMapBio $_{14}$ & $\operatorname{LogMapC}_{14}$ \\
\hline ELK & 0.07 & 0.05 & 17 & 0.11 & 0.12 & 0.45 & 0.13 & 0.08 \\
\hline HERMIT & 0.6 & 0.5 & 5.93 & 0.6 & 1.03 & 1.12 & 0.7 & 0.48 \\
\hline KONCLUDE & 0.54 & 0.7 & 0.61 & 0.51 & 0.57 & 0.79 & 0.57 & 0.6 \\
\hline $\begin{array}{l}\text { PELLET } \\
\end{array}$ & 2.45 & 2.22 & ERR & ERR & ERR & ERR & ERR & ERR \\
\hline & \multicolumn{2}{|c|}{\begin{tabular}{|l|l} 
MaasMatch $_{13}$ &
\end{tabular}} & $\overline{\text { ODGOMS }}_{13}$ & Reference $_{14}$ & RSDLWB $_{14}$ & $\overline{\text { WeSeE}_{13}}$ & 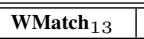 & YAM++ 13 \\
\hline ELK & \multicolumn{2}{|c|}{6.67} & 0.12 & 0.05 & 0.08 & 0.28 & 0.11 & 0.06 \\
\hline HERMIT & \multicolumn{2}{|c|}{5.6} & 0.91 & 0.58 & 0.88 & 4 & 0.84 & 0.8 \\
\hline KONCLUDE & \multicolumn{2}{|c|}{0.6} & 0.55 & 0.53 & 0.55 & 0.62 & 0.67 & 0.56 \\
\hline PELLET & \multicolumn{2}{|c|}{\begin{tabular}{|l|l} 
ERR \\
\end{tabular}} & ERR & 2.11 & ERR & ERR & ERR & 2.32 \\
\hline
\end{tabular}

Table 6

Classification times (s), largebio-small dataset with selected mapping sets.

\begin{tabular}{|c|c|c|c|c|c|c|c|c|c|c|c|c|c|}
\hline FMA-NCI & $\mathbf{A M L}_{14}$ & \multicolumn{2}{|c|}{ GOMMA $_{13}$} & IAMA $_{13}$ & \multicolumn{2}{|c|}{ LogMapBio $_{14}$} & \multicolumn{2}{|c|}{ MaasMatch $_{14}$} & \multicolumn{2}{|c|}{ OMReasoner $_{14}$} & \multicolumn{2}{|c|}{ Reference $_{13}$} & YAM++ 13 \\
\hline ELK & 0.12 & \multicolumn{2}{|c|}{1.17} & 0.1 & \multicolumn{2}{|c|}{0.19} & \multicolumn{2}{|c|}{0.21} & \multicolumn{2}{|c|}{0.1} & \multicolumn{2}{|c|}{1.07} & 0.11 \\
\hline HERMIT & 16 & \multicolumn{2}{|c|}{21} & 15 & \multicolumn{2}{|c|}{18} & \multicolumn{2}{|c|}{3.58} & \multicolumn{2}{|c|}{18} & \multicolumn{2}{|c|}{53} & 18 \\
\hline KONCLUDE & 7.6 & \multicolumn{2}{|c|}{7.45} & 7.44 & \multicolumn{2}{|c|}{8.25} & \multicolumn{2}{|c|}{1.3} & \multicolumn{2}{|c|}{9.52} & \multicolumn{2}{|c|}{6.01} & 9.81 \\
\hline PELLET & 19 & \multicolumn{2}{|c|}{17} & 17 & & & & & & & & 11 & 22 \\
\hline FMA-SNOMED & AML $_{14}$ & GON & $\mathbf{I A}_{13}$ & IAM & $\log 1$ & pBio $_{14}$ & Maa & $\operatorname{Iatch}_{14}$ & OMR & soner $_{14}$ & & erence $_{13}$ & YAM++ 13 \\
\hline ELK & 0.78 & & & & & 78 & & 22 & & 57 & & 0.89 & 0.58 \\
\hline HERMIT & 11 & & & & & 11 & & 86 & & & & 3.75 & 5.23 \\
\hline KONCLUDE & 4.82 & & & & & 63 & & 77 & & 99 & & 3.37 & 3.83 \\
\hline PELLET & 2,119 & & & & & DUT & & UT & & 2 & & /OUT & T/OUT \\
\hline SNOME & O-NCI & AML $_{14}$ & GON & $\mathbf{M A}_{13}$ & IAMA $_{13}$ & LogMa & Bio $_{14}$ & OMRea & oner $_{14}$ & Refere & $e_{13}$ & YAM++ & \\
\hline ELK & & 4.26 & & 58 & 3.09 & & & & & 4.3 & & 3.41 & \\
\hline HERM & & T/OUT & & 3 & 57 & $\mathrm{~T} / \mathrm{O}$ & & & & 4.9 & & T/OU' & \\
\hline KONCI & UDE & OOM & & 7 & 16 & $\mathrm{OC}$ & & & & 19 & & $\mathrm{OOM}$ & \\
\hline PELLE & & T/OUT & & UT & T/OUT & $\mathrm{T} / \mathrm{O}$ & & & UT & $\mathrm{T} / \mathrm{O}$ & & T/OUT & \\
\hline
\end{tabular}

Table 7

Classification times (s), largebio-big dataset with selected mapping sets.

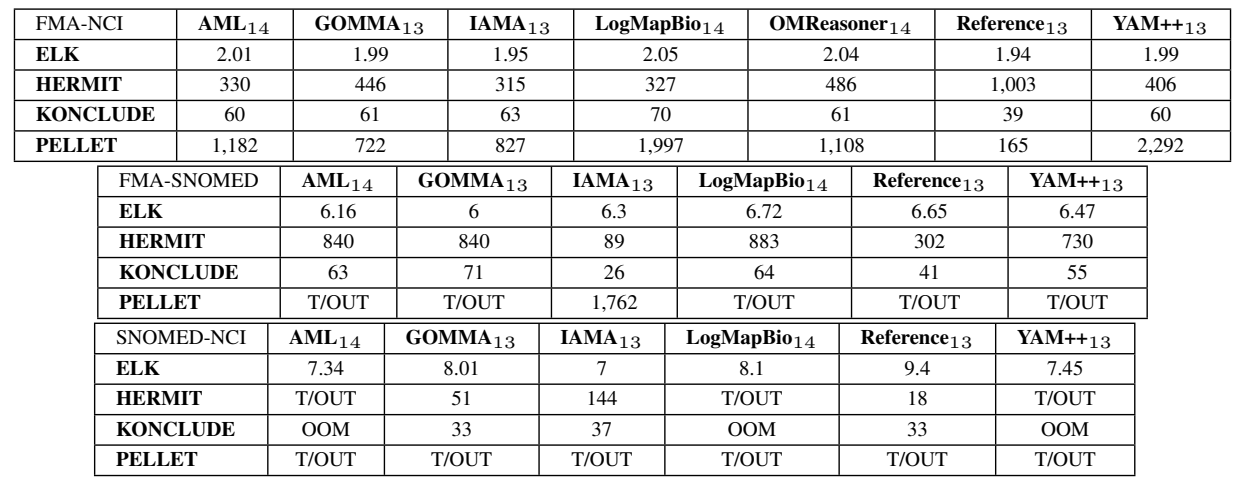

Table 8

Classification times (s), library dataset with selected mapping sets.

\begin{tabular}{|c|c|c|c|c|c|c|c|c|}
\hline & $\mathbf{A M L}_{13}$ & $\mathbf{A M L}_{14}$ & Hertuda $_{13}$ & IAMA $_{13}$ & $\operatorname{LogMap}_{13}$ & $\operatorname{LogMapC}_{14}$ & MaasMatch $_{14}$ & ODGOMS $_{13}$ \\
\hline ELK & 13 & 0.47 & 35 & 0.06 & 1.06 & 0.07 & 9.76 & 0.32 \\
\hline HERMIT & 1,059 & 16 & 68 & 1.03 & 1.68 & 0.9 & 37 & 1.91 \\
\hline KONCLUDE & 5.81 & 2.28 & 17 & 1.13 & 1.72 & 0.78 & 2.3 & 1.14 \\
\hline PELLET & T/OUT & 7.79 & T/OUT & 0.22 & 1.08 & 0.17 & T/OUT & 1.69 \\
\hline & \multicolumn{2}{|c|}{$\mid$ Reference $_{14}$} & RSDLWB ${ }_{14}$ & StringsAuto $_{13}$ & Xmap $_{14}$ & $\overline{\text { XmapGen }_{13}}$ & XmapSig $_{13}$ & YAM++13 \\
\hline ELK & \multicolumn{2}{|c|}{0.1} & 0.06 & 0.07 & 29 & T/OUT & 0.16 & 2.09 \\
\hline HERMIT & \multicolumn{2}{|c|}{0.86} & 0.94 & 0.86 & 56 & T/OUT & 1.57 & 7.5 \\
\hline KONCLUDE & \multicolumn{2}{|c|}{0.86} & 1.2 & 0.81 & 8.06 & 59 & 1.77 & 2.22 \\
\hline PELLET & \multicolumn{2}{|c|}{0.29} & 0.22 & 0.2 & T/OUT & T/OUT & 1 & 87 \\
\hline
\end{tabular}


Table 9

Classification times (s), conference dataset with selected mapping sets

\begin{tabular}{|c|c|c|c|c|c|c|c|c|c|c|c|c|c|}
\hline \multicolumn{2}{|c|}{ CMT-IASTED } & $\mathbf{A M L}_{14}$ & AMLbk $_{1}$ & \multicolumn{2}{|c|}{ MaasMatch $_{14}$} & \multicolumn{2}{|c|}{ Reference $_{14}$} & \multicolumn{2}{|c|}{ XMapGen $_{13}$} & \multicolumn{2}{|c|}{ XMapSig $_{13}$} & YAM++ & ++13 \\
\hline ELK & & 0.02 & 0.02 & \multicolumn{2}{|c|}{0.02} & \multicolumn{2}{|c|}{0.02} & \multicolumn{2}{|c|}{0} & \multicolumn{2}{|c|}{0.07} & 0.02 & 02 \\
\hline \multicolumn{2}{|c|}{ HERMIT } & 0.11 & 0.18 & \multicolumn{2}{|c|}{0.14} & \multicolumn{2}{|c|}{0.13} & \multicolumn{2}{|c|}{0.12} & \multicolumn{2}{|c|}{0.12} & 0.12 & 12 \\
\hline \multicolumn{2}{|c|}{ KONCLUDE } & 0.1 & 0.18 & \multicolumn{2}{|c|}{0.09} & \multicolumn{2}{|c|}{0.08} & \multicolumn{2}{|c|}{0.17} & \multicolumn{2}{|c|}{0.08} & 0.08 & \\
\hline \multicolumn{2}{|c|}{ PELLET } & 2.58 & 4.11 & \multicolumn{2}{|c|}{ ERR } & \multicolumn{2}{|c|}{2.64} & \multicolumn{2}{|c|}{2.6} & \multicolumn{2}{|c|}{2.58} & 2.57 & \\
\hline & \multicolumn{2}{|c|}{ CONFOF-IASTED } & AML $_{14}$ & AMLbk $_{13}$ & \multicolumn{2}{|c|}{ Reference $_{14}$} & \multicolumn{2}{|c|}{ XMapGen $_{13}$} & \multicolumn{2}{|c|}{$\frac{1}{\text { XMapSig }_{13}}$} & \multicolumn{2}{|c|}{ YAM++13 } & \\
\hline \multicolumn{3}{|c|}{ ELK } & 0.01 & 0 & \multicolumn{2}{|c|}{0.12} & \multicolumn{2}{|c|}{0} & & 01 & & & \\
\hline & HERM & & 0.22 & 0.26 & & 0.24 & & 0.22 & & 21 & & & \\
\hline & KONCI & JDE & 0.12 & 0.2 & & 0.07 & & 0.18 & & .13 & & & \\
\hline & PELLE & & 3.28 & 3.48 & & 3.36 & & 4.26 & & 28 & & & \\
\hline & EDAS & ASTED & $\overline{\text { AML }_{14}}$ & AMLbk $_{13}$ & Refe & -ence ${ }_{14}$ & XMap & $\overline{\text { Gen }_{13}}$ & XMap & $\overline{\operatorname{Sig}_{13}}$ & YAM & & \\
\hline & ELK & & 0.03 & 0.01 & & .02 & & 01 & ( & & 0. & & \\
\hline & HERI & & 0.14 & 0.14 & & .16 & & 26 & 0 . & & 0 . & & \\
\hline & KONC & UDE & 0.18 & 0.16 & & .08 & & 22 & 0. & & 0 . & & \\
\hline & PELL & & 2.11 & 2.3 & & .08 & & 76 & 2. & & & & \\
\hline & & CONFER & ENCE-IASTEI & D:MaasMatch & & TASTED-S & GKDD: & $\overline{\text { AOTL }}_{14}$ & $\overline{\text { IAS }}$ & [ED-SIG & KDD:M & asMatch & $\mathrm{h}_{14}$ \\
\hline ELK & & & 0.06 & & & & 0.02 & & & & 0.04 & & \\
\hline HERM & & & T/OUT & & & & 13 & & & & 0.58 & & \\
\hline KONC & LUDE & & 0.36 & & & & 0.25 & & & & 0.09 & & \\
\hline PELLI & & & 7.05 & & & & 16 & & & & 2.86 & & \\
\hline
\end{tabular}

Table 10

Justification extraction in the anatomy dataset with MaasMatch $_{13}$

(a) Unsatisfiabilites

\begin{tabular}{|c|c|c|c|c|c|c|}
\hline Reasoner & Class.(s) & \#Unsat & 1Just.(s) & \%1JustOK & 50Just.(s) & \%50JustOK \\
\hline ELK & 6.67 & 5,972 & 68 & 100 & 8,542 & 100 \\
\hline $\mathbf{E L K}_{\text {trace }}$ & 6.67 & 5,972 & 769 & 0 & 6,001 & 0 \\
\hline HermiT & 5.6 & 5,972 & 19 & 100 & 2,726 & 100 \\
\hline
\end{tabular}

(b) Conservativity violations

\begin{tabular}{|l|c|c|c|c|c|c|}
\hline Reasoner & Class.(s) & \#Viol & 1Just.(s) & \% 1JustOK & 50Just.(s) & \%50JustOK \\
\hline \hline ELK & 6.67 & 64,580 & 1.17 & 100 & 140 & 100 \\
\hline ELK $_{\text {trace }}$ & 6.67 & 64,580 & 15 & 0 & 120 & 0 \\
\hline HermiT & 5.6 & 64,580 & 0.2 & 100 & 47 & 100 \\
\hline
\end{tabular}

Table 11

Justification extraction in the anatomy dataset with $\mathrm{WeSeE}_{13}$

(a) Unsatisfiabilites

\begin{tabular}{|l|c|c|c|c|c|c|}
\hline Reasoner & Class.(s) & \#Unsat & 1Just.(s) & \%1JustOK & 50Just.(s) & \%50JustOK \\
\hline \hline ELK & 0.28 & 2,015 & 7.65 & 100 & 51 & 100 \\
\hline ELK $_{\text {trace }}$ & 0.28 & 2,015 & 1.19 & 0 & 160 & 98 \\
\hline HermiT & 4 & 2,015 & 5.98 & 100 & 497 & 100 \\
\hline
\end{tabular}

(b) Conservativity violations

\begin{tabular}{|l|c|c|c|c|c|c|}
\hline Reasoner & Class.(s) & \#Viol & 1Just.(s) & \% 1JustOK & 50Just.(s) & \%50JustOK \\
\hline \hline ELK & 0.28 & 7,298 & 0.12 & 100 & 43 & 100 \\
\hline ELK $_{\text {trace }}$ & 0.28 & 7,298 & 0.01 & 16 & 4.66 & 98 \\
\hline HermiT & 4 & 7,298 & 0.08 & 100 & 71 & 98 \\
\hline
\end{tabular}


Table 12

Justification extraction in the conference dataset (CONFERENCE-EDAS) with MaasMatch ${ }_{13}$

(a) Unsatisfiabilites

\begin{tabular}{|l|c|c|c|c|c|c|}
\hline Reasoner & Class.(s) & \#Unsat & 1Just.(s) & \% 1JustOK & 50Just.(s) & \%50JustOK \\
\hline \hline ELK & 0 & 78 & 5.67 & 100 & 583 & 100 \\
\hline ELK & 0 & 78 & 0.44 & 0 & 271 & 100 \\
\hline Hermi & 0.02 & 93 & 2.77 & 100 & 455 & 100 \\
\hline Pellet & 0.03 & 93 & 1.92 & 100 & 277 & 100 \\
\hline
\end{tabular}

(b) Conservativity violations

\begin{tabular}{|l|c|c|c|c|c|c|}
\hline Reasoner & Class.(s) & \#Viol & 1Just.(s) & \%1JustOK & 50Just.(s) & \%50JustOK \\
\hline \hline ELK & 0 & 7 & 0.11 & 100 & 10 & 100 \\
\hline ELK $_{\text {trace }}$ & 0 & 7 & 0.01 & 0 & 4.79 & 100 \\
\hline HermiT & 0.02 & 8 & 0.04 & 100 & 11 & 100 \\
\hline Pellet & 0.03 & 8 & 0.03 & 100 & 6.99 & 100 \\
\hline
\end{tabular}

Table 13

Justification extraction in the conference dataset (CONFERENCE-EKAW) with MaasMatch 14

(a) Unsatisfiabilites

\begin{tabular}{|c|c|c|c|c|c|c|}
\hline Reasoner & Class.(s) & \#Unsat & 1Just.(s) & \%1JustOK & 50Just.(s) & \%50JustOK \\
\hline ELK & 0.02 & 54 & 37 & 100 & 1,037 & 100 \\
\hline $\mathbf{E L K}_{\text {trace }}$ & 0.02 & 54 & 1.52 & 0 & 694 & 98 \\
\hline HermiT & 0.01 & 63 & 3.3 & 100 & 296 & 100 \\
\hline Pellet & 0.01 & 63 & 62 & 98 & 2,569 & 16 \\
\hline
\end{tabular}

(b) Conservativity violations

\begin{tabular}{|c|c|c|c|c|c|c|}
\hline Reasoner & Class.(s) & \#Viol & 1Just.(s) & \%1JustOK & 50Just.(s) & \%50JustOK \\
\hline ELK & 0.02 & 114 & 0.46 & 100 & 29 & 100 \\
\hline $\mathbf{E L K}_{\text {trace }}$ & 0.02 & 114 & 0.05 & 12 & 7.48 & 98 \\
\hline HermiT & 0.01 & 115 & 0.04 & 100 & 4.1 & 100 \\
\hline Pellet & 0.01 & 115 & 0.02 & 100 & 21 & 68 \\
\hline
\end{tabular}

Table 14

Justification extraction in the conference dataset (CONFOF-EDAS) with XMapGen 13

(a) Unsatisfiabilites

\begin{tabular}{|l|c|c|c|c|c|c|}
\hline Reasoner & Class.(s) & \#Unsat & 1Just.(s) & \%1JustOK & 50Just.(s) & \%50JustOK \\
\hline \hline ELK & 0 & 23 & 2.7 & 100 & 23 & 100 \\
\hline ELK $_{\text {trace }}$ & 0 & 23 & 0.29 & 0 & 8 & 100 \\
\hline HermiT & 0.02 & 31 & 2.26 & 100 & 6,702 & 97 \\
\hline Pellet & 0.01 & 31 & 1.22 & 100 & 6,169 & 97 \\
\hline
\end{tabular}

(b) Conservativity violations

\begin{tabular}{|l|c|c|c|c|c|c|}
\hline Reasoner & Class.(s) & \#Viol & 1Just.(s) & \%1JustOK & 50Just.(s) & \%50JustOK \\
\hline \hline ELK & 0 & 6 & 0.03 & 100 & 1.36 & 100 \\
\hline ELK $_{\text {trace }}$ & 0 & 6 & 0.01 & 0 & 0.49 & 100 \\
\hline HermiT & 0.02 & 6 & 0.02 & 100 & 27 & 100 \\
\hline Pellet & 0.01 & 6 & 0.01 & 100 & 13 & 100 \\
\hline
\end{tabular}


Table 15

Justification extraction in the conference dataset (CONFOF-SIGKDD) with OOTL $_{14}$

(a) Unsatisfiabilites

\begin{tabular}{|c|c|c|c|c|c|c|}
\hline Reasoner & Class.(s) & \#Unsat & 1Just.(s) & \% 1JustOK & 50Just.(s) & \%50JustOK \\
\hline ELK & 0.01 & 61 & 25 & 100 & 2,355 & 100 \\
\hline $\mathbf{E L K}_{\text {trace }}$ & 0.01 & 61 & 1.53 & 0 & 1,104 & 90 \\
\hline HermiT & 0 & 61 & 1.9 & 100 & 1,054 & 100 \\
\hline Pellet & 0.01 & 61 & 1.01 & 100 & 1,015 & 100 \\
\hline
\end{tabular}

(b) Conservativity violations

\begin{tabular}{|l|c|c|c|c|c|c|}
\hline Reasoner & Class.(s) & \#Viol & 1Just.(s) & \%1JustOK & 50Just.(s) & \%50JustOK \\
\hline \hline ELK & 0.01 & 2 & 0.3 & 100 & 1,500 & 50 \\
\hline ELK $_{\text {trace }}$ & 0.01 & 2 & 0.03 & 0 & 60 & 50 \\
\hline HermiT & 0 & 2 & 0.04 & 100 & 1,500 & 50 \\
\hline Pellet & 0.01 & 2 & 0.01 & 100 & 1,500 & 50 \\
\hline
\end{tabular}

Table 16

Justification extraction in FMA-NCI (largebio-big dataset) with Reference 13

(a) Unsatisfiabilites

\begin{tabular}{|c|c|c|c|c|c|c|}
\hline Reasoner & Class.(s) & \#Unsat & 1Just.(s) & \%1JustOK & 50Just.(s) & \%50JustOK \\
\hline ELK & 1.94 & 30,494 & 41 & 100 & 20,294 & 92 \\
\hline $\mathbf{E L K}_{\text {trace }}$ & 1.94 & 30,494 & 2.58 & 0 & 1,587 & 82 \\
\hline Hermi T & 1,003 & 30,590 & 49 & 100 & 13,836 & 92 \\
\hline Pellet & 165 & 30,590 & 42 & 100 & 30,878 & 80 \\
\hline
\end{tabular}

(b) Conservativity violations

\begin{tabular}{|c|c|c|c|c|c|c|}
\hline Reasoner & Class.(s) & \#Viol & 1Just.(s) & \%1JustOK & 50Just.(s) & \%50JustOK \\
\hline ELK & 1.94 & 20,710 & 0.88 & 100 & 429 & 86 \\
\hline $\mathbf{E L K}_{\text {trace }}$ & 1.94 & 20,710 & 0.01 & 36 & 22 & 86 \\
\hline HermiT & 1,003 & 20,814 & 1.06 & 100 & 422 & 88 \\
\hline
\end{tabular}

Table 17

Justification extraction in FMA-NCI (largebio-big dataset) with GOMMA 13

(a) Unsatisfiabilites

\begin{tabular}{|l|c|c|c|c|c|c|}
\hline Reasoner & Class.(s) & \#Unsat & 1Just.(s) & \%1JustOK & 50Just.(s) & \%50JustOK \\
\hline \hline ELK & 1.99 & 5,561 & 45 & 100 & 153 & 100 \\
\hline ELK $_{\text {trace }}$ & 1.99 & 5,561 & 2.28 & 0 & 55 & 100 \\
\hline HermiT & 446 & 5,574 & 76 & 100 & 700 & 100 \\
\hline Pellet & 722 & 5,574 & 62 & 100 & 1,333 & 100 \\
\hline
\end{tabular}

(b) Conservativity violations

\begin{tabular}{|c|c|c|c|c|c|c|}
\hline Reasoner & Class.(s) & \#Viol & 1Just.(s) & \%1JustOK & 50Just.(s) & \%50JustOK \\
\hline ELK & 1.99 & 14,187 & 0.85 & 100 & 3.37 & 100 \\
\hline $\mathbf{E L K}_{\text {trace }}$ & 1.99 & 14,187 & 0.03 & 68 & 1.61 & 100 \\
\hline HermiT & 446 & 14,635 & 1.17 & 100 & 13 & 100 \\
\hline
\end{tabular}


Table 18

Justification extraction in FMA-SNOMED (largebio-big dataset) with Reference ${ }_{13}$

(a) Unsatisfiabilites

\begin{tabular}{|c|c|c|c|c|c|c|}
\hline Reasoner & Class.(s) & \#Unsat & 1Just.(s) & \%1JustOK & 50Just.(s) & \%50JustOK \\
\hline ELK & 6.65 & 78,482 & 75 & 100 & 20,709 & 88 \\
\hline $\mathbf{E L K}_{\text {trace }}$ & 6.65 & 78,482 & 7.3 & 0 & 2,666 & 78 \\
\hline HermiT & 302 & 78,482 & 102 & 100 & 4,169 & 98 \\
\hline
\end{tabular}

(b) Conservativity violations

\begin{tabular}{|c|c|c|c|c|c|c|}
\hline Reasoner & Class.(s) & \#Viol & 1Just.(s) & \%1JustOK & 50Just.(s) & \%50JustOK \\
\hline ELK & 6.65 & 187,121 & 1.7 & 100 & 76 & 100 \\
\hline $\mathbf{E L K}_{\text {trace }}$ & 6.65 & 187,121 & 0.02 & 30 & 29 & 94 \\
\hline HermiT & 302 & 187,121 & 3 & 100 & 55 & 100 \\
\hline
\end{tabular}

Table 19

Justification extraction in FMA-SNOMED (largebio-big dataset) with YAM++13

(a) Unsatisfiabilites

\begin{tabular}{|c|c|c|c|c|c|c|}
\hline Reasoner & Class.(s) & \#Unsat & 1Just.(s) & \%1JustOK & 50Just.(s) & \%50JustOK \\
\hline ELK & 6.47 & 57,074 & 67 & 100 & 12,052 & 96 \\
\hline $\mathbf{E L K}_{\text {trace }}$ & 6.47 & 57,074 & 6.83 & 0 & 2,098 & 82 \\
\hline HermiT & 730 & 57,074 & 179 & 100 & 5,001 & 100 \\
\hline
\end{tabular}

(b) Conservativity violations

\begin{tabular}{|c|c|c|c|c|c|c|}
\hline Reasoner & Class.(s) & \#Viol & 1Just.(s) & \%1JustOK & 50Just.(s) & \%50JustOK \\
\hline ELK & 6.47 & 117,316 & 1.74 & 100 & 440 & 86 \\
\hline $\mathbf{E L K}_{\text {trace }}$ & 6.47 & 117,316 & 0.03 & 40 & 31 & 86 \\
\hline HermiT & 730 & 119,236 & 4.44 & 100 & 269 & 94 \\
\hline
\end{tabular}

Table 20

Justification extraction in SNOMED-NCI (largebio-big dataset) with GOMMA $_{13}$

(a) Unsatisfiabilites

\begin{tabular}{|c|c|c|c|c|c|c|}
\hline Reasoner & Class.(s) & \#Unsat & 1Just.(s) & \%1JustOK & 50Just.(s) & \%50JustOK \\
\hline ELK & 8.01 & 127,850 & 90 & 100 & 20,741 & 90 \\
\hline $\mathbf{E L K}_{\text {trace }}$ & 8.01 & 127,850 & 9.37 & 0 & 1,746 & 86 \\
\hline HermiT & 51 & 131,222 & 94 & 100 & 15,983 & 84 \\
\hline
\end{tabular}

(b) Conservativity violations

\begin{tabular}{|l|c|c|c|c|c|c|}
\hline Reasoner & Class.(s) & \#Viol & 1Just.(s) & \%1JustOK & 50Just.(s) & \%50JustOK \\
\hline \hline ELK & 8.01 & 151,488 & 2.43 & 100 & 250 & 96 \\
\hline ELK $_{\text {trace }}$ & 8.01 & 151,488 & 0.01 & 4 & 22 & 92 \\
\hline
\end{tabular}


Table 21

Justification extraction in SNOMED-NCI (largebio-big dataset) with LogMapBio 14

(a) Unsatisfiabilites

\begin{tabular}{|l|c|c|c|c|c|c|}
\hline Reasoner & Class.(s) & \#Unsat & 1Just.(s) & \% 1JustOK & 50Just.(s) & \%50JustOK \\
\hline \hline ELK & 8.1 & 37 & 123 & 100 & 4,387 & 100 \\
\hline ELK $_{\text {trace }}$ & 8.1 & 37 & 8.4 & 0 & 349 & 100 \\
\hline
\end{tabular}

(b) Conservativity violations

\begin{tabular}{|l|c|c|c|c|c|c|}
\hline Reasoner & Class.(s) & \#Viol & 1Just.(s) & \% 1JustOK & 50Just.(s) & \%50JustOK \\
\hline \hline ELK & 8.1 & 439,887 & 3.58 & 100 & 706 & 86 \\
\hline ELK $_{\text {trace }}$ & 8.1 & 439,887 & 0.02 & 14 & 31 & 100 \\
\hline
\end{tabular}

Table 22

Justification extraction in SNOMED-NCI (largebio-big dataset) with Reference 13

(a) Unsatisfiabilites

\begin{tabular}{|l|c|c|c|c|c|c|}
\hline Reasoner & Class.(s) & \#Unsat & 1Just.(s) & \%1JustOK & 50Just.(s) & \%50JustOK \\
\hline \hline ELK & 9.4 & 158,645 & 99 & 100 & 17,293 & 92 \\
\hline ELK $_{\text {trace }}$ & 9.4 & 158,645 & 13 & 0 & 1,674 & 90 \\
\hline HermiT & 18 & 161,202 & 83 & 100 & 15,126 & 90 \\
\hline
\end{tabular}

(b) Conservativity violations

\begin{tabular}{|l|c|c|c|c|c|c|}
\hline Reasoner & Class.(s) & \#Viol & 1Just.(s) & \%1JustOK & 50Just.(s) & \%50JustOK \\
\hline \hline ELK & 9.4 & 576,736 & 3.03 & 100 & 332 & 92 \\
\hline ELK $_{\text {trace }}$ & 9.4 & 576,736 & 0.02 & 0 & 44 & 88 \\
\hline
\end{tabular}


Table 23

Justification extraction in FMA-NCI (largebio-small dataset) with Reference ${ }_{13}$

(a) Unsatisfiabilites

\begin{tabular}{|l|c|c|c|c|c|c|}
\hline Reasoner & Class.(s) & \#Unsat & 1Just.(s) & \%1JustOK & 50Just.(s) & \%50JustOK \\
\hline \hline ELK & 1.07 & 4,401 & 19 & 100 & 25,929 & 84 \\
\hline ELK $_{\text {trace }}$ & 1.07 & 4,401 & 1.53 & 0 & 1,864 & 78 \\
\hline HermiT & 53 & 4,402 & 5.84 & 100 & 7,338 & 96 \\
\hline Pellet & 11 & 4,402 & 7.3 & 100 & 12,609 & 94 \\
\hline
\end{tabular}

(b) Conservativity violations

\begin{tabular}{|l|c|c|c|c|c|c|}
\hline Reasoner & Class.(s) & \#Viol & 1Just.(s) & \% 1JustOK & 50Just.(s) & \%50JustOK \\
\hline \hline ELK & 1.07 & 21,296 & 0.15 & 100 & 253 & 94 \\
\hline ELK $_{\text {trace }}$ & 1.07 & 21,296 & 0.02 & 36 & 21 & 88 \\
\hline HermiT & 53 & 21,400 & 0.07 & 100 & 309 & 90 \\
\hline
\end{tabular}

Table 24

Justification extraction in FMA-NCI (largebio-small dataset) with LogMapBio 14

(a) Unsatisfiabilites

\begin{tabular}{|l|c|c|c|c|c|c|}
\hline Reasoner & Class.(s) & \#Unsat & 1Just.(s) & \% 1JustOK & 50Just.(s) & \%50JustOK \\
\hline \hline ELK & 0.19 & 0 & 0 & 0 & 0 & 0 \\
\hline ELK $_{\text {trace }}$ & 0.19 & 0 & 0 & 0 & 0 & 0 \\
\hline HermiT & 18 & 467 & 16 & 100 & 3,777 & 100 \\
\hline Pellet & 29 & 467 & 11 & 100 & 2,288 & 100 \\
\hline
\end{tabular}

(b) Conservativity violations

\begin{tabular}{|c|c|c|c|c|c|c|}
\hline Reasoner & Class.(s) & \#Viol & 1Just.(s) & \%1JustOK & 50Just.(s) & \%50JustOK \\
\hline ELK & 0.19 & 26,657 & 0.14 & 100 & 61 & 98 \\
\hline $\mathbf{E L K}_{\text {trace }}$ & 0.19 & 26,657 & 0.04 & 74 & 3.14 & 98 \\
\hline HermiT & 18 & 26,603 & 0.08 & 100 & 1.44 & 100 \\
\hline
\end{tabular}

Table 25

Justification extraction in FMA-SNOMED (largebio-small dataset) with GOMMA 13

(a) Unsatisfiabilites

\begin{tabular}{|c|c|c|c|c|c|c|}
\hline Reasoner & Class.(s) & \#Unsat & 1Just.(s) & \%1JustOK & 50Just.(s) & \%50JustOK \\
\hline ELK & 0.75 & 2,058 & 16 & 100 & 50,919 & 70 \\
\hline $\mathbf{E L K}_{\text {trace }}$ & 0.75 & 2,058 & 1.09 & 0 & 2,855 & 62 \\
\hline HermiT & 9.33 & 2,058 & 9.42 & 100 & 22,909 & 86 \\
\hline Pellet & 273 & 2,058 & 6.47 & 100 & 15,963 & 90 \\
\hline
\end{tabular}

(b) Conservativity violations

\begin{tabular}{|c|c|c|c|c|c|c|}
\hline Reasoner & Class.(s) & \#Viol & 1Just.(s) & \%1JustOK & 50Just.(s) & \%50JustOK \\
\hline ELK & 0.75 & 18,425 & 0.29 & 100 & 73 & 98 \\
\hline $\mathbf{E L K}_{\text {trace }}$ & 0.75 & 18,425 & 0.06 & 88 & 8.68 & 94 \\
\hline HermiT & 9.33 & 18,425 & 0.14 & 100 & 67 & 98 \\
\hline
\end{tabular}


Table 26

Justification extraction in FMA-SNOMED (largebio-small dataset) with IAMA 13

(a) Unsatisfiabilites

\begin{tabular}{|c|c|c|c|c|c|c|}
\hline Reasoner & Class.(s) & \#Unsat & 1Just.(s) & \%1JustOK & 50Just.(s) & \%50JustOK \\
\hline ELK & 0.49 & 22,925 & 8.7 & 100 & 10,839 & 94 \\
\hline $\mathbf{E L K}_{\text {trace }}$ & 0.49 & 22,925 & 0.77 & 0 & 1,040 & 88 \\
\hline HermiT & 0.54 & 22,925 & 5.78 & 100 & 151 & 100 \\
\hline Pellet & 1.73 & 22,925 & 4.96 & 100 & 66 & 100 \\
\hline
\end{tabular}

(b) Conservativity violations

\begin{tabular}{|l|c|c|c|c|c|c|}
\hline Reasoner & Class.(s) & \#Viol & 1Just.(s) & \%1JustOK & 50Just.(s) & \%50JustOK \\
\hline \hline ELK & 0.49 & 2,270 & 0.23 & 100 & 51 & 100 \\
\hline ELK $_{\text {trace }}$ & 0.49 & 2,270 & 0.01 & 2 & 26 & 90 \\
\hline HermiT & 0.54 & 2,270 & 0.13 & 100 & 12 & 100 \\
\hline
\end{tabular}

Table 27

Justification extraction in FMA-SNOMED (largebio-small dataset) with MaasMatch 14

(a) Unsatisfiabilites

\begin{tabular}{|c|c|c|c|c|c|c|}
\hline Reasoner & Class.(s) & \#Unsat & 1Just.(s) & \%1JustOK & 50Just.(s) & \%50JustOK \\
\hline ELK & 4.22 & 21,946 & 40 & 100 & 10,267 & 98 \\
\hline $\mathbf{E L K}_{\text {trace }}$ & 4.22 & 21,946 & 5.82 & 0 & 3,626 & 78 \\
\hline HermiT & 2.86 & 21,946 & 25 & 100 & 3,219 & 100 \\
\hline
\end{tabular}

(b) Conservativity violations

\begin{tabular}{|c|c|c|c|c|c|c|}
\hline Reasoner & Class.(s) & \#Viol & 1Just.(s) & \%1JustOK & 50Just.(s) & \%50JustOK \\
\hline ELK & 4.22 & 717,697 & 0.89 & 100 & 289 & 100 \\
\hline $\mathbf{E L K}_{\text {trace }}$ & 4.22 & 717,697 & 0.04 & 2 & 91 & 58 \\
\hline HermiT & 2.86 & 697,459 & 0.51 & 100 & 188 & 98 \\
\hline
\end{tabular}


Table 28

Justification extraction in SNOMED-NCI (largebiosmall dataset) with LogMapBio 14

(a) Unsatisfiabilites

\begin{tabular}{|l|c|c|c|c|c|c|}
\hline Reasoner & Class.(s) & \#Unsat & 1Just.(s) & \%1JustOK & 50Just.(s) & \%50JustOK \\
\hline \hline ELK & 4.8 & 23 & 210 & 87 & 10,030 & 87 \\
\hline ELK $_{\text {trace }}$ & 4.8 & 23 & 5.3 & 0 & 585 & 100 \\
\hline
\end{tabular}

(b) Conservativity violations

\begin{tabular}{|l|c|c|c|c|c|c|}
\hline Reasoner & Class.(s) & \#Viol & 1Just.(s) & \%1JustOK & 50Just.(s) & \%50JustOK \\
\hline \hline ELK & 4.8 & 750,226 & 2.87 & 100 & 733 & 86 \\
\hline ELK $_{\text {trace }}$ & 4.8 & 750,226 & 0.03 & 10 & 34 & 98 \\
\hline
\end{tabular}

Table 29

Justification extraction in SNOMED-NCI (largebiosmall dataset) with Reference 13

(a) Unsatisfiabilites

\begin{tabular}{|c|c|c|c|c|c|c|}
\hline Reasoner & Class.(s) & \#Unsat & 1Just.(s) & \%1JustOK & 50Just.(s) & \%50JustOK \\
\hline ELK & 4.36 & 66,832 & 74 & 100 & 6,983 & 98 \\
\hline $\mathbf{E L K}_{\text {trace }}$ & 4.36 & 66,832 & 6.23 & 0 & 1,417 & 94 \\
\hline HermiT & 4.94 & 69,218 & 58 & 100 & 6,913 & 88 \\
\hline
\end{tabular}

(b) Conservativity violations

\begin{tabular}{|l|c|c|c|c|c|c|}
\hline Reasoner & Class.(s) & \#Viol & 1Just.(s) & \%1JustOK & 50Just.(s) & \%50JustOK \\
\hline \hline ELK & 4.36 & 576,736 & 1.53 & 100 & 266 & 96 \\
\hline ELK $_{\text {trace }}$ & 4.36 & 576,736 & 0.02 & 0 & 42 & 86 \\
\hline
\end{tabular}

Table 30

Justification extraction in SNOMED-NCI (largebiosmall dataset) with OMReasoner 14

(a) Unsatisfiabilites

\begin{tabular}{|c|c|c|c|c|c|c|}
\hline Reasoner & Class.(s) & \#Unsat & 1Just.(s) & \%1JustOK & 50Just.(s) & \%50JustOK \\
\hline ELK & 2.95 & 35,568 & 38 & 100 & 9,501 & 96 \\
\hline $\mathbf{E L K}_{\text {trace }}$ & 2.95 & 35,568 & 3.74 & 0 & 1,517 & 86 \\
\hline HermiT & 74 & 39,942 & 38 & 100 & 24,054 & 88 \\
\hline
\end{tabular}

(b) Conservativity violations

\begin{tabular}{|l|c|c|c|c|c|c|}
\hline Reasoner & Class.(s) & \#Viol & 1Just.(s) & \%1JustOK & 50Just.(s) & \%50JustOK \\
\hline \hline ELK & 2.95 & 62,783 & 1.03 & 100 & 201 & 96 \\
\hline ELK $_{\text {trace }}$ & 2.95 & 62,783 & 0.01 & 12 & 25 & 90 \\
\hline
\end{tabular}


Table 31

Justification extraction in the library dataset

(a) Conservativity violations with $\mathrm{AML}_{13}$

\begin{tabular}{|l|c|c|c|c|c|c|}
\hline Reasoner & Class.(s) & \#Viol & 1Just.(s) & \%1JustOK & 50Just.(s) & \%50JustOK \\
\hline \hline ELK & 13 & $15,708,616$ & 35 & 66 & 2,794 & 14 \\
\hline HermiT & 1,059 & $15,708,616$ & 3.58 & 100 & 315 & 100 \\
\hline
\end{tabular}

(b) Conservativity violations with Hertuda 13

\begin{tabular}{|l|c|c|c|c|c|c|}
\hline Reasoner & Class.(s) & \#Viol & 1Just.(s) & \% 1JustOK & 50Just.(s) & \%50JustOK \\
\hline \hline ELK & 35 & $26,782,359$ & 37 & 62 & 2,878 & 12 \\
\hline HermiT & 68 & $26,782,359$ & 2.91 & 100 & 414 & 98 \\
\hline
\end{tabular}

(c) Conservativity violations with $\mathrm{XMap}_{14}$

\begin{tabular}{|l|c|c|c|c|c|c|}
\hline Reasoner & Class.(s) & \#Viol & 1Just.(s) & \%1JustOK & 50Just.(s) & \%50JustOK \\
\hline \hline ELK & 29 & $24,507,254$ & 33 & 62 & 2,641 & 22 \\
\hline HermiT & 56 & $24,507,254$ & 2.07 & 100 & 303 & 98 \\
\hline
\end{tabular}

